\title{
Diferencias entre discursos colectivos (verbales) $e$ individuales (escritos) al hacer demostraciones en geometría: una explicación a partir del sistema de normas
}

Differences between Collective (Verbal) and Individual (Written) Discourses when Proving in Geometry: An Explanation from the Norms System

Oscar Molina ${ }^{1}$

Luis Pino-Fan²

\begin{abstract}
Resumen. Con el fin de involucrar a estudiantes de secundaria en un curso de geometría plana centrado en la práctica de demostrar, fue replicada una innovación que se ha venido desarrollando en un curso de nivel universitario y cuyos efectos han sido interpretados como exitosos. Uno de los resultados de dicha réplica constituye un hecho no esperado por parte del grupo investigativo y de los profesores del curso que participaron en la experiencia: no existe correspondencia entre las producciones verbales de los alumnos -cuando se involucran en la construcción colectiva de la demostración de una conjetura formulada por la clase producto de la solución de un problema- y las producciones autónomas que realizan al reportar por escrito la demostración previamente construida de manera colectiva. Esta situación problemática es explicada mediante un dispositivo analítico que articula constructos tales como situaciones instruccionales, contrato didáctico, normas sociomatemáticas y discurso reflexivo, a partir de los cuales se estudia la interacción en el aula y el rol del profesor en ella.
\end{abstract}

Fecha de recepción: 25 de abril de 2017. Fecha de aceptación: 9 de febrero de 2018.

${ }^{1}$ Departamento de Matemáticas, Facultad de Ciencia y Tecnología, Universidad Pedagógica Nacional (Bogotá, Colombia). Doctorando en Educación Matemática, Universidad de Los Lagos (Chile). ojmolina@ pedagogica.edu.co

2 Departamento de Ciencias Exactas, Universidad de Los Lagos (Chile). luis.pino@ulagos.cl 
Palabras clave: interacción en el aula; discurso reflexivo; contrato didáctico; normas sociomatemáticas; demostración.

\begin{abstract}
To involve students of a high school geometry course in the proving process, it was replicated a methodological innovation that has been implemented in a university geometry course and whose effects have been interpreted as successful. One result of this replica shows an unexpected fact by both the research group and teachers involved in the experience: there is a mismatch between students' verbal productions when they engage in collective construction of the proof of a conjecture formulated by whole class as product of the solution of a problem, and the productions that the students autonomously perform when they must report in writing the proof that was previously constructed collectively. This problematic issue is explained by an analytical device that articulates constructs such as instructional situations, didactic contract, sociomathematical norms and reflexive discourse, from which the interaction in the classroom and the teacher's role in it are studied.
\end{abstract}

Keywords: interaction in the classroom; reflective discourse; didactic contract; sociomathematical norms, proof.

\title{
INTRODUCCIÓN
}

Los educadores en matemáticas tienen como desafío importante lograr que sus estudiantes comprendan el papel de la demostración en la actividad matemática (Hanna \& de Villiers, 2012). En este sentido, el grupo de investigación Aprendizaje y Enseñanza de la Geometría de la Universidad Pedagógica Nacional (Bogotá, Colombia), del cual el primer autor es miembro, ha desarrollado una trayectoria investigativa centrada principalmente en una perspectiva sociocultural de la enseñanza y el aprendizaje, a través de la cual se ha generado una innovación en el aula para cursos universitarios de geometría euclidiana, que intenta involucrar a los estudiantes en la actividad demostrativa (Perry, Samper, Molina, Camargo \& Echeverry, 2012; Samper, Camargo, Molina \& Perry, 2013; Molina, Samper \& Perry, 2015; Samper, Perry, Camargo, Sáenz-Ludlow \& Molina, 2015). En tal innovación, la fuente de donde se toma el contenido que se estudia no proviene de los libros ni del profesor. Una cantidad considerable de los enunciados que 
se demuestran los formula la comunidad de la clase, en calidad de conjeturas provenientes de las producciones de los alumnos al resolver problemas (abiertos de conjeturación) $)^{3}$ propuestos por el docente. Asimismo, los estudiantes, con el apoyo del profesor, analizan las demostraciones que se hacen en el curso. En ocasiones, algunos hechos geométricos (postulados y teoremas) o definiciones de objetos ${ }^{4}$ son incorporados de manera legítima al sistema teórico, porque se advierte su necesidad para poder usarlos en la demostración que se está construyendo.

Se destacan tres elementos sobre los cuales recae el esfuerzo didáctico innovador con el propósito de generar un entorno favorable para aprender a demostrar: (i) Los problemas abiertos de conjeturación; (ii) El uso de un Entorno de Geometría Dinámica (EGD) como artefacto que posibilita la exploración de situaciones y, en consecuencia, brinda recursos para solucionar los problemas propuestos; y (iii) La interrelación social en la clase, compuesta por tres interacciones específicas: trabajo de los estudiantes (a través del cual autónomamente realizan tareas propuestas por el profesor, como resolver un problema o escribir una demostración previamente construida de manera colectiva), conversación instruccional (a través de la cual el docente gestiona la socialización de las producciones de los alumnos con miras a guiar a la comunidad en la construcción de significados compartidos y en la organización colectiva de las ideas que encontraron para producir las demostraciones) y conversación matemática (i.e., un diálogo entre el profesor y los estudiantes, o entre estos, sobre un tema matemático específico en el cual las ideas se comunican, comentan y critican).

En el marco del proyecto Geometría: Vía al Razonamiento Científico (desarrollado en 2015 y 2016), ${ }^{5}$ el grupo de investigación mencionado hizo una réplica de tal innovación para cursos de geometría básica de primaria y secundaria, con el propósito de ver la posibilidad de que los estudiantes se involucraran en prácticas argumentativas (incluyendo la demostración). En ese marco, uno de

3 En un problema abierto de conjeturación su enunciado no revela una respuesta y pide explícitamente establecer como solución una conjetura (i.e., proposición condicional $p \rightarrow q$ ); en geometría, consiste en la descripción de una configuración y la solicitud de una proposición relativa a las relaciones entre sus elementos o propiedades (Baccaglini-Frank \& Mariotti, 2010).

4 Los objetos geométricos y su respectiva definición son introducidos para satisfacer una necesidad manifiesta de su uso en el marco de los procesos de resolución de problemas o demostración. El estudio de la definición, por un lado, parte de la imagen conceptual que los estudiantes tienen del objeto y, por otro, precisa de un análisis centrado en el papel de cada condición dentro de la definición.

5 Proyecto financiado por el Centro de Investigaciones Universidad Pedagógica Nacional, Bogotá-Colombia (CIUP). 
los escenarios fue un curso de grado noveno (alumnos de entre 14 y 15 años) de un colegio público ubicado en Bogotá, Colombia, en el cual fue implementada una secuencia didáctica compuesta por tres problemas que abordan dos objetos relativos a la relación de equidistancia (la circunferencia y la mediatriz de un segmento como lugares geométricos). Análisis de los registros de información - específicamente, de la conversación matemática que tiene lugar cuando la clase está inmersa en la demostración colectiva de una conjetura surgida como solución de uno de los problemas y del trabajo de los estudiantes, que consiste en la escritura autónoma de la demostración previamente construida de manera colectiva- ponen en evidencia las diferencias en las producciones de los alumnos en ambas interacciones, lo cual dista de lo que espera el profesor (y el grupo de investigación).

El presente artículo se propone explicar la diferencia que acabamos de describir empleando un dispositivo analítico que articula tres aspectos centrales, el segundo y el tercero fuertemente influenciados por el primero: (i) el rol que juega el discurso en el aula de clase para apoyar el desarrollo matemático de los estudiantes (Cobb, Boufi, McClain \& Whitenack, 1997), en este caso respecto a una demostración válida desde un punto de vista matemático; (ii), el rol de la interacción en el aula entre profesores y alumnos, específicamente en relación a cláusulas del contrato didáctico; y (iii), las normas socio-matemáticas que circulan en el aula (Jones \& Herbst, 2012).

El artículo está estructurado de la siguiente manera: primero presenta los referentes teóricos que constan de una conceptualización sobre el discurso reflexivo (Cobb, Boufi, McClain \& Whitenack, 1997) y algunos constructos de teorías relativas a la interacción en el aula; se expone cómo estos referentes pueden complementarse entre sí para construir el dispositivo analítico usado. Enseguida presenta, grosso modo, la metodología del estudio; luego ilustra el análisis con algunos momentos de interacción en el aula. Para terminar, incluye algunas reflexiones.

\section{REFERENTES TEÓRICOS}

En esta sección presentamos la conceptualización referida a los constructos demostración, discurso e interacción en el aula, centrales para precisar el dispositivo con el cual hacemos el análisis. En el primer apartado establecemos la relación entre argumentación y demostración. 


\section{DEMOSTRACIÓN}

En correspondencia con varios autores de la escuela italiana (Mariotti, Bartolini Bussi, Boero, Ferri \& Garuti, 1997; Boero, 1999; Boero, Douek, Morselli \& Pedemonte, 2010), en este texto se considera la demostración (o prueba matemática) como un tipo especial de argumentación. Tales autores, que asumen una postura sociocultural, entienden por argumentación el proceso colectivo o individual que, de acuerdo con reglas compartidas, apunta a una conclusión aceptable acerca de la veracidad o falsedad de una aserción o acción, o a la búsqueda de la toma de una decisión (Krummheuer, 1995). El discurso oral o escrito producto del proceso de argumentación se compone de argumentos (Krummheuer, 1995; Toulmin, 2003). Específicamente, tal como lo propone Toulmin (2003), un argumento está compuesto por tres elementos básicos: La aserción (A), o punto de vista pronunciado por alguien; los datos (D) que soportan la aserción A cuando es desafiada; y la garantía (G), que presenta la incidencia de los datos $D$ en la aserción A, cuando se desafía de qué forma los datos pueden ser conectados a la aserción A. La garantía G puede ser expresada mediante un principio o una regla general que autoriza el paso de los datos $\mathrm{D}$ a la aserción C. Desde esta perspectiva, una demostración es una argumentación compuesta por una secuencia de argumentos conectados lógicamente (soportados por reglas de inferencia lógico-deductivas), cuyas garantías son definiciones, postulados o teoremas de un sistema teórico (Stylianides, 2007); usualmente el producto (discurso) de este tipo de argumentación es denominado también demostración o prueba.

\section{DISCURSO EN EL AULA}

A principios de la década de los 90 surgió una tendencia investigativa en la comunidad de educadores en matemáticas enfocada en analizar el rol que tiene el discurso en el aula de clase, para apoyar el desarrollo matemático de los estudiantes que participan en él. Dentro de esta tendencia se alude al cambio en el discurso o al constructo discurso reflexivo (Cobb, Boufi, McClain \& Whitenack, 1997), esto es, un discurso de clase que se caracteriza por ser emergente y evidenciar un cambio respecto a uno previo, producto de la discusión sobre el objeto en torno al cual se da el discurso. Cobb y colaboradores (1997) conjeturan que la participación de los alumnos en una actividad de clase que posibilite un 
discurso reflexivo provoca la matematización progresiva de los discursos previamente producidos por ellos; esta actividad se concreta con la mediación del profesor, que brinda oportunidad de reflexión sobre actividades previas y, de esa manera, las convierte en objeto de estudio. Para los casos que acá se reportan, el discurso (verbal y escrito) que circula en las clases está relacionado con la actividad que tiene lugar cuando se demuestra el enunciado de un teorema de geometría plana euclidiana.

Para lograr el objetivo que nos hemos propuesto, nos interesa dar seguimiento a la emergencia de discursos reflexivos por parte de los alumnos, y la labor del profesor para que ello suceda. Por ejemplo, un indicador de cambio en el discurso ocurre cuando, en la construcción colectiva de una demostración, ante preguntas hechas por el docente sobre los elementos que componen un argumento (según el modelo de Toulmin, 2003), los estudiantes proveen, según el caso, aserciones, garantías y datos válidos en el sistema teórico compartido por la comunidad de la clase, en lugar de sus percepciones empíricas. Otro ejemplo de discurso reflexivo se presenta cuando los alumnos escriben un texto de una demostración cuyos argumentos fueron construidos colectivamente en una actividad previa; para ello, necesitan reflexionar sobre la argumentación realizada para poder sistematizar los pasos (argumentos) presentes en dicho trabajo previo, aludiendo a ellos no aisladamente sino en el marco de cadenas deductivas que los relacionan. ${ }^{6}$

A fin de precisar otros discursos reflexivos que se esperan de los estudiantes, detallamos a continuación tipos de discursos que se corresponden con las diferentes maneras en que se puede presentar una demostración para comunicarla: (i) Es posible comunicar una demostración de manera tal que solo exponga los pasos estructurales (estructura modular) ${ }^{7}$ que la componen -en adelante, pasos estructurales de Nivel 1-; y (ii) Al comunicar una demostración se presenta un encadenamiento fino de todas las aserciones (no solamente las estructurales), explicitando las garantías de cada aserción y los datos empleados para usar tales garantías; en otras palabras, se desarrolla con cierto detalle la estructura modular de la demostración -en adelante, pasos de Nivel 2- (Mejia-Ramos, Fuller, Weber, Rhoads \& Samkoff,

\footnotetext{
${ }^{6}$ Otra forma de un discurso reflexivo puede darse cuando los estudiantes explícitamente discuten sobre constructos como argumentación, demostración o formatos para presentarla. En el curso objeto de estudio, este caso emergió durante la interacción.

7 Son aquellos pasos que permiten tener una idea general de la demostración, sin necesidad de precisar los detalles de deducción de cada paso argumental.
} 
2012). Samper y Plazas (2017) aluden a formatos especiales para presentar una demostración, que se corresponden con esta clasificación: por un lado, usan el formato Núcleos-Pilares, mediante el cual se presentan demostraciones que exponen sus pasos estructurales (de Nivel 1), siendo los Núcleos las respectivas aserciones y los prilares las garantías correspondientes; por otro lado, aluden al formato Afirmación-Garantía para presentar demostraciones de Nivel 2. Con el propósito de ilustrar la diferencia entre estos dos tipos de discursos, consideremos como ejemplo una demostración del siguiente enunciado: Si los puntos A y B pertenecen a una circunferencia, entonces el centro de la circunferencia pertenece a la mediatriz de $\overline{A B}$. La Tabla 1 presenta una demostración con el encadenamiento fino de todos los pasos (argumentos) que la componen (Afirmación-Garantía); están sombreadas las celdas de los pasos que componen la estructura modular de la demostración (Núcleos-Pilares).

Con la descripción anterior se tiene un contexto para precisar otros indicadores de discurso reflexivo por parte de los estudiantes: luego de la elaboración colectiva de la demostración se espera, por ejemplo, que para el momento de su escritura autónoma, los alumnos aludan a sus pasos estructurales (de Nivel 1; ver pasos sombreados de la Tabla 1), esto es, a una estructura que contiene los Núcleos y Pilares de la demostración (en adelante, se llamará indicador de cambio de discurso D2). Ahora bien, cada uno de tales pasos está constituido por argumentos (presuntamente abordados en la interacción colectiva) que desarrollan tales ideas estructurales. Se espera, entonces, que los estudiantes también aludan a dichos argumentos, que apelen a una estructura que contiene todas las Afirmaciones y Garantías (de Nivel 2) de la demostración (indicador de cambio de discurso D3). Este es un tipo de discurso más "fino" que D2, el cual permite inferir o desarrollar los Núcleos de la demostración. Por supuesto estos cambios de discurso, tal como se explicó, pueden indicar un discurso reflexivo, pues implican hacer una sistematización de la reflexión sobre los pasos que se espera emerjan durante la construcción colectiva de la demostración. 
Tabla 1. Demostración por Afirmación-Garantía y por Núcleo-Pilares.

\begin{tabular}{|c|c|c|}
\hline & Afirmación (Núcleo) & Garantía $^{8}$ (Pilar) \\
\hline 1. & Puntos $A$ y $B$ & Dado \\
\hline 2. & $A, B \in \bigodot_{C, r}$ & Dado \\
\hline 3. & $A C=r$ & D. Circunferencia ${ }^{9}(2)$ \\
\hline 4. & $B C=r$ & D. Circunferencia (2) \\
\hline 5. & $A C=C B$ & Propiedad transitiva de la igualdad (3 y 4 ) \\
\hline 6. & $\overline{A C} \cong \overline{C B}$ & D. Segmentos congruentes (5) \\
\hline \multicolumn{3}{|c|}{ Caso 1. Si C no pertenece a $\overline{A B}$} \\
\hline 7. & Sea $D$ el punto medio de $\overline{A B}$ & Por construcción auxiliar (1) \\
\hline 8. & D equidista de $\mathrm{A}$ y $\mathrm{B}$ & D. Punto medio (7) \\
\hline 9. & $A D=D B$ & D. Equidistancia (8) \\
\hline 10. & $\overline{A D} \cong \overline{B D}$ & D. Segmentos congruentes (9) \\
\hline 11. & Sean $\triangle A C D$ y $\triangle B D C$ & Por construcción auxiliar $(1,2)$ \\
\hline 12. & $\overline{C D} \cong \overline{C D}$ & Propiedad reflexiva de la igualdad \\
\hline 13. & $\triangle A C D \cong \triangle B D C$ & P. $\operatorname{LLL}(6,10,12)$ \\
\hline 14. & $\angle C D A \cong \angle C D B$ & D. Triángulos congruentes (13) \\
\hline 15. & $\angle C D A$ y $\angle C D B$ son par lineal & Por representación (11) \\
\hline 16. & $m \angle C D A+m \angle C D B=180$ & P. Par lineal ${ }^{10}(15)$ \\
\hline 17. & $m \angle C D A=m \angle C D B$ & D. Congruencia de ángulos (14) \\
\hline 18. & $m \angle C D A+m \angle C D A=180$ & Sustitución $(16,17)$ \\
\hline 19. & $2 m \angle C D A=180 ; m \angle C D A=90$ & Propiedad de los reales (18) \\
\hline 20. & $\angle C D A$ y $\angle C D B$ rectos & Definición de ángulos rectos $(19,17)$ \\
\hline 21. & $\overleftrightarrow{C D} \perp \overline{A B}$ & D. Rectas perpendiculares (20) \\
\hline 22. & 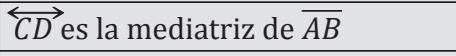 & D. Mediatriz de un segmento 11 \\
\hline 23. & C pertenece a la mediatriz de $\overline{A B}$ & D. Mediatriz (22) \\
\hline
\end{tabular}

${ }^{8}$ Las letras D, P y T significan, respectivamente, Definición, Postulado y Teorema. Los números entre paréntesis aluden a los pasos donde están las afirmaciones necesarias para utilizar como datos en la garantía correspondiente, y con base en estos (datos y garantía) inferir deductivamente la nueva aserción.

${ }^{9}$ Definición de Circunferencia: Dados un punto $\mathrm{O}$ en un plano y un número positivo $\mathrm{r}$, una circunferencia es el conjunto de puntos del mismo plano que equidistan de $\mathrm{O}$ una distancia $\mathrm{r}$.

10 Postulado Par Lineal: Dados dos ángulos que son par lineal, entonces la suma de sus medidas es 180.

11 Definición de mediatriz de un segmento: Una recta es mediatriz de un segmento si es perpendicular al segmento por su punto medio. 


\begin{tabular}{l|l|l}
\hline \multicolumn{3}{l}{ Caso 2. Si C pertenece al $\overline{A B}$} \\
\hline 24. & C punto medio de $\overline{A B}$ & D. Punto medio (5) \\
\hline 25. & C pertenece a la mediatriz de $\overline{A B}$ & D. Mediatriz (19) \\
\hline
\end{tabular}

\section{INTERACCIÓN EN EL AULA}

El discurso reflexivo no solo favorece una posición crítica del alumno sobre el objeto de estudio, sino también la interacción social en la clase, particularmente entre estudiantes y profesor. Asumiendo la posición de Jones y Herbst (2012), usamos perspectivas teóricas que aluden a tal interacción desde una aproximación social, focalizándonos en el rol del docente en la enseñanza de la demostración. Nos interesa precisar si propicia la participación de los alumnos durante la práctica argumentativa (lo cual se indicaría con la construcción colectiva de una demostración), y si favorece un cambio en el discurso pronunciado en dicha participación (indicado, por ejemplo, en la escritura de una demostración válida basada en la previamente construida). Para dar cuenta del rol mencionado, Jones y Herbst (2012) aluden a la posibilidad de relacionar dos perspectivas teóricas para lograr el objetivo: Las normas sociomatemáticas (Yackel \& Cobb, 1996) y la Teoría de los intercambios instruccionales (Jones \& Herbst, 2012).

\section{NORMAS SOCIOMATEMÁTICAS}

La teoría de las normas sociomatemáticas provee una manera de interpretar las aulas de matemáticas con el objetivo de explicar cómo los alumnos desarrollan creencias y valores matemáticos y, en consecuencia, cómo se vuelven intelectualmente autónomos en matemáticas (Yackel \& Cobb, 1996). La teoría propone que, cuando se intenta involucrar a los estudiantes en la demostración, el hecho de que deban justificar ${ }^{12}$ sus afirmaciones se constituye en una norma social de la clase. En ese contexto, en su papel de representantes de la disciplina en el aula, los profesores podrían promover normas sociomatemáticas asociadas con esa norma social. En particular, los docentes deberían promover lo que consideran una justificación matemática apropiada. Martin, McCrone, Bower y Dindyal

12 Entiéndase justificar como la acción a través de la cual se precisan los datos que soportan una aserción y la garantía que conecta tales datos con dicha aserción. 
(2005) sostienen que, con el fin de crear un clima en el aula en el cual los alumnos hagan conjeturas, proporcionen justificaciones, construyan cadenas de razonamiento y presenten una demostración comprensible para otros, el profesor debe participar en un diálogo que sitúe la responsabilidad de las argumentaciones en ellos, analizar sus argumentos y dirigirlos a medida que argumentan. En este artículo nos proponemos precisar si ciertas normas socio-matemáticas (e.g., toda afirmación debe argumentarse en el marco del sistema teórico dado en la clase $-\mathrm{NS}_{1}{ }^{-}$, una demostración se corresponde con una cadena de razonamiento válida en matemáticas $-\mathrm{NS}_{3}-$, el lenguaje utilizado se corresponde con un lenguaje geométrico $-\mathrm{NS}_{3}{ }^{-}$,) emergen durante la interacción en el aula y si son promovidas por el docente.

\section{TEORÍA DE LOS INTERCAMBIOS INSTRUCCIONALES}

Jones y Herbst (2012) precisan dos elementos centrales de esta teoría: el contrato didáctico y las variadas escalas de tiempo en la actividad de la clase. El primer elemento alude a la posibilidad de un contrato mediante el cual, el profesor adquiere la responsabilidad de atender a los estudiantes como aprendices de matemáticas y de precisar las matemáticas que necesitan ser enseñadas. Asimismo, los alumnos asumen el contrato de aprender las matemáticas enseñadas y comportarse de acuerdo con ellas. El segundo elemento refiere la posibilidad de analizar el trabajo de instrucción del maestro a partir de dos aspectos que conviven en escalas de tiempo diferentes: hay un trabajo de gestión de momento a momento (durante intercambios de discursos de los estudiantes entre ellos y con el profesor, por ejemplo) y un trabajo con los objetos matemáticos de conocimiento que existen en escalas de tiempo mayor (objeto que se desea abordar a la luz del plan de estudios anual, por ejemplo). Desde esta perspectiva, el docente necesita gestionar intercambios entre las actividades de escala de tiempo pequeño y los objetos de conocimiento que están en una escala mayor, esto debido a que las actividades de momento a momento le sirven para implementar o crear instancias de los objetos matemáticos a gran escala. Recíprocamente, los objetos de conocimiento sirven para evaluar las actividades de momento a momento.

En esa perspectiva, se proponen dos formas básicas que favorecen dichos intercambios: negociación de tareas nuevas y situación instruccional. En la primera forma, un profesor necesita tanto manejar tareas que son completamente 
nuevas para los estudiantes como involucrarlos en tales tareas y decidir (o negociar) cómo aplicar en ellas el contrato didáctico. En particular, esta decisión o negociación incluye identificar qué aspectos de la tarea refieren al conocimiento matemático en juego y cuáles del trabajo de los alumnos en esa tarea evidencian que están adquiriendo o han adquirido el conocimiento. La segunda forma se enmarca en un intercambio, de acuerdo con las cláusulas del contrato previamente constituidas (que han sido usuales para la clase); en este caso, el trabajo del docente no consiste en procurar la identificación de las matemáticas en la tarea (como sucedía por medio de la tarea nueva), sino procurar la identificación de la situación misma, actuando de acuerdo con el contrato que constituye dicha situación. Para el caso que acá se aborda (curso de geometría de grado noveno), la situación instruccional relativa a la construcción de una demostración de un hecho geométrico estaba caracterizada por cláusulas del contrato didáctico usuales en las clases tradicionales de geometría; esto es, el profesor tenía la responsabilidad de exponer las aserciones de cada uno de los pasos de la demostración y, eventualmente, solicitar a los estudiantes las garantías correspondientes. Por su parte, los alumnos tenían la responsabilidad de seguir la exposición del docente, proveer las garantías cuando él las solicitaba e ir escribiendo en su cuaderno los pasos argumentales de la demostración a la vez que el maestro los iba presentando. En lo que respecta a la Tarea Nueva (consistente en la construcción colectiva de la demostración y su posterior escritura autónoma), en la Tabla 2 exponemos el contrato didáctico que debe surgir como nuevo para que la actividad de los estudiantes se diferencie de aquella que tenía en la situación instruccional descrita.

\section{RELACIÓN ENTRE LAS TEORÍAS}

La teoría de los intercambios instruccionales provee una estructura para articular los aspectos teóricos comentados, pues se compone de tres elementos centrales que permiten analizar (o diseñar) una clase en la cual se pretende construir una demostración ${ }^{13}$ (Jones \& Herbst, 2012): contrato didáctico, actividad de clase en

${ }^{13}$ La teoría de los intercambios instruccionales alude a una relación con base en un modelo de interacción en el aula denominado hacer pruebas. Conlleva a un sistema de normas (sociales y socio-matemáticas) que puede ser útil para diseñar o analizar la interacción en el aula con relación a la demostración. 
variadas escalas de tiempo y formas de intercambio. En el presente artículo nos valemos de la relación entre tales elementos y aspectos del discurso para generar un dispositivo que permita analizar los momentos de clase. Así, se conciben por lo menos tres relaciones: (i) Entre las normas sociomatemáticas de la clase y el contrato didáctico (tanto las cláusulas para el profesor como para el estudiante); (ii) Entre las formas de intercambio descritas y el contrato didáctico del docente en particular; y (iii) Entre las ideas respecto al discurso, propuesta por Cobb et al. (1997), y las normas sociomatemáticas.

Con respecto a la relación $i$, vale decir que consideramos posible que el uso reiterado de ciertas cláusulas del contrato didáctico ocasione la instauración de normas sociomatemáticas, las cuales regulan los aspectos específicos de las discusiones matemáticas de los estudiantes (Godino, Font, Wilhelmi \& Castro, 2009), para este caso, de aquellos que tienen lugar en la construcción colectiva de una demostración de un hecho geométrico. En este sentido, la Tabla 2 destaca las principales cláusulas del contrato didáctico para los alumnos y el profesor y las normas sociomatemáticas que se le podrían asociar (i.e., las que se podrían instaurar si tales cláusulas se cumplen reiteradamente). Entre corchetes se usan las letras M, E y P: la M indica cláusulas relacionadas con las matemáticas; la E, aquellas relativas principalmente a las responsabilidades de aprendizaje por parte de los alumnos; y la $\mathrm{P}$, las correspondientes principalmente a las responsabilidades de enseñanza por parte del docente.

En cuanto a la relación ii, cada forma de intercambio es caracterizada por cláusulas específicas del contrato didáctico (Jones \& Herbst, 2012). La Tabla 2 expone las cláusulas presentes en las formas de intercambios que tendrían lugar en las clases que se analizan; las normas sociomatemáticas respectivas deberían estar presentes en cualquiera de las dos formas de intercambio. Finalmente, con respecto a la relación iii, es razonable pensar que el cumplimiento reiterado de cláusulas del contrato didáctico cause la producción de discursos por parte de los estudiantes como los descritos en una sección anterior, los cuales, a su vez, son indicadores de la instauración (o cumplimiento) de las normas sociomatemáticas asociadas. La Tabla 2 muestra los discursos esperados y las normas sociomatemáticas que se espera circulen en la clase que sirvió de escenario para este reporte. Los elementos presentados en dicha tabla se convierten en indicadores preliminares mediante los cuales fueron analizados los momentos de tal clase. En la sección "Descripción y análisis de momentos" se establece la relación entre las normas y los tipos de discurso emergentes. 
Tabla 2. Relación entre formas de intercambio, contrato didáctico, normas sociomatemáticas y discursos. ${ }^{14} 15$

\begin{tabular}{|c|c|c|c|c|}
\hline \multirow{2}{*}{$\begin{array}{c}\text { Formas } \\
\text { instruccionales } \\
\text { Tareas Nuevas } \\
\end{array}$} & \multicolumn{2}{|c|}{ Contrato didáctico } & \multirow{2}{*}{$\begin{array}{l}\text { Normas socio- } \\
\text { matemáticas } \\
\text { De clase }\end{array}$} & \multirow{2}{*}{$\begin{array}{l}\text { Tipos de } \\
\text { discurso de } \\
\text { estudiantes }\end{array}$} \\
\hline & Profesor & Estudiante & & \\
\hline $\begin{array}{l}\text { Construir } \\
\text { conjuntamente } \\
\text { (con } \\
\text { participación de } \\
\text { "todos" -profesor } \\
\text { y estudiantes-) } \\
\text { una } \\
\text { demostración de } \\
\text { un hecho } \\
\text { geométrico. (S) } \\
\text { Producir } \\
\text { autónomamente } \\
\text { el texto escrito } \\
\text { de la } \\
\text { demostración de } \\
\text { un hecho } \\
\text { geométrico } \\
\text { empleando el } \\
\text { formato } \\
\text { Afirmación- } \\
\text { Garantía. }\end{array}$ & $\begin{array}{l}\mathrm{CDP}_{1} \text { Debe } \\
\text { favorecer la } \\
\text { participación de } \\
\text { los estudiantes } \\
\text { mediante } \\
\text { preguntas de } \\
\text { indagación (por } \\
\text { qué). [P] } \\
\mathrm{CDP}_{2} \text { Debe } \\
\text { analizar los } \\
\text { argumentos de } \\
\text { los estudiantes. } \\
\text { [M] } \\
\text { CDP }_{3} \text { Debe } \\
\text { orientar a los } \\
\text { estudiantes, a } \\
\text { medida que ellos } \\
\text { argumentan, y } \\
\text { redirigirlos } \\
\text { cuando las } \\
\text { garantías, } \\
\text { argumentos, o } \\
\text { cadenas de } \\
\text { argumentación } \\
\text { no son válidos. [P] } \\
\text { CDP }_{4} \text { Debe } \\
\text { destacar aquellos } \\
\text { pasos } \\
\text { estructurales (de } \\
\text { Nivel 1) de la } \\
\text { demostración. [M] }\end{array}$ & $\begin{array}{l}\mathrm{CDE}_{1} \text { Debe } \\
\text { argumentar } \\
\text { afirmaciones } \\
\text { (propias o no) en } \\
\text { el marco del } \\
\text { sistema teórico } \\
\text { dado en la clase, } \\
\text { i.e., usando } \\
\text { garantías } \\
\text { provenientes de } \\
\text { la geometría } \\
\text { plana euclidiana. } \\
\text { [M] } \\
\mathrm{CDE}_{2} \text { Debe } \\
\text { proveer } \\
\text { demostraciones } \\
\text { de acuerdo con } \\
\text { una cadena de } \\
\text { argumentos. [M] } \\
\mathrm{CDE}_{3} \text { Debe } \\
\text { responder todo } \\
\text { cuestionamiento } \\
\text { que se le haga. } \\
\text { [E] } \\
\mathrm{CDE}_{4} \text { Debe } \\
\text { procurar } \\
\text { participar } \\
\text { autónomamente } \\
\text { en las } \\
\text { actividades de } \\
\text { clase. [E] }\end{array}$ & $\begin{array}{l}\mathrm{NS}_{1} \text { Toda } \\
\text { afirmación debe } \\
\text { argumentarse en } \\
\text { el marco del } \\
\text { sistema teórico } \\
\text { (de la geometría } \\
\text { euclidiana) dado } \\
\text { en la clase. } \\
\text { NS }_{2} \text { Una } \\
\text { demostración es } \\
\text { una cadena } \\
\text { (lógica-deductiva) } \\
\text { de razonamiento } \\
\text { válida en las } \\
\text { matemáticas. Se } \\
\text { presenta en el } \\
\text { formato Aserción- } \\
\text { Garantía. } \\
\text { NS } \text { (Norma }_{3} \\
\text { previamente } \\
\text { instaurada que } \\
\text { debe estar } \\
\text { presente en todo } \\
\text { momento de la } \\
\text { clase). Se debe } \\
\text { emplear un } \\
\text { leguaje } \\
\text { geométrico } \\
\text { válido }{ }^{15} \text { tanto en } \\
\text { lo verbal como en } \\
\text { lo escrito. }\end{array}$ & $\begin{array}{l}\mathrm{D}_{1} \text { Alusión } \\
\text { explícita a } \\
\text { garantías } \\
\text { teóricas y datos } \\
\text { para justificar } \\
\text { aserciones. } \\
\mathrm{D}_{2} \text { Alusión a } \\
\text { pasos } \\
\text { estructurales de } \\
\text { Nivel } 1 \text { (Núcleos } \\
\text { y Pilares) en la } \\
\text { demostración. } \\
\mathrm{D}_{3} \text { Alusión a } \\
\text { pasos de Nivel } 2 \\
\text { (Afirmación y } \\
\text { Garantía) en la } \\
\text { demostración. } \\
\mathrm{D}_{4} \text { (Discurso } \\
\text { reflexivo) } \\
\text { Sistematización } \\
\text { de la } \\
\text { demostración } \\
\text { hecha } \\
\text { colectivamente, } \\
\text { presentada en } \\
\text { un texto escrito } \\
\text { que usa el } \\
\text { formato } \\
\text { Afirmación- } \\
\text { Garantía. }\end{array}$ \\
\hline
\end{tabular}

14 Los acrónimos (en negrita) se usaron como códigos para analizar las interacciones de la clase.

15 Para la clase, un lenguaje geométrico válido implica utilizar la simbología formal para los objetos y relaciones de la geometría y teoría de conjuntos, y usar la terminología formal para los mismos. Gran parte de ellos fueron introducidos en el trascurso de los cursos previos de geometría. 


\section{CONTEXTO GENERAL DE LAS SESIONES DE CLASES Y DISEÑO EXPERIMENTAL}

La descripción y análisis de los episodios de clase corresponden a sucesos ocurridos en dos sesiones de clase de un curso de secundaria de geometría plana euclidiana, conformado por 34 estudiantes entre 13 y 15 años, del Instituto Pedagógico Nacional, ubicado en Bogotá, Colombia. En dicho curso se realizó un experimento de enseñanza (Steffe \& Thompson, 2000) mediante el cual se pretendía que los alumnos se involucraran en un ambiente de actividad demostrativa. El equipo de investigación diseñó e implementó una secuencia de problemas cuyo objeto de estudio se centró en las conexiones entre la mediatriz y la circunferencia a partir de la relación de equidistancia. Por limitaciones de espacio, en este escrito se expone parte del análisis a posteriori del experimento, concentrado en episodios donde (i) Estudiantes y profesor se focalizan en construir colectivamente la demostración de una conjetura; y (ii) Los alumnos deben construir autónomamente el texto escrito de tal demostración. Vale decir que previa a la implementación de la secuencia, hubo una etapa de instrucción mediante la cual se pretendía que los estudiantes se familiarizaran con la aproximación metodológica para la enseñanza (Perry, Samper, Molina, Camargo \& Echeverry, 2012) descrita en la introducción. La secuencia fue implementada por dos profesores del instituto, uno el titular del curso (P1) y otra, una profesora (P2) que apoyó la actividad del docente titular. Los episodios de clase utilizados para el análisis se concentran en la actividad matemática que gira en torno al siguiente problema (último de la secuencia constituida por tres problemas):

Dados dos puntos A y B: i) ¿Existe una circunferencia que los contiene? ii) ¿Existe otra? ¿Existen más? ${ }^{16}$ iii) Si tiene sentido, responda las siguientes preguntas con base en las respuestas a los items i y ii, ¿dónde se encuentra el centro de la(s) circunferencia(s) que contiene(n) los puntos $A$ y $B$ ?

El abordaje de tal problema tomó cuatro sesiones de clase en total. Durante las dos primeras, con ayuda de GeoGebra, los estudiantes organizados por parejas exploraron la situación expuesta en el problema y proveyeron una conjetura como solución. En la tercera se llevó a cabo una conversación

\footnotetext{
16 No hubo grupo de estudiantes que hayan encontrado solamente una circunferencia.
} 
instruccional mediante la cual P1 presentó cada una de las conjeturas producidas por los alumnos; expuso primero las que contenían más falencias hasta mostrar, al final, la mejor formulada. Los asuntos tenidos en cuenta para cualificarlas fueron el contenido geométrico mismo, el lenguaje utilizado, correspondencia con el procedimiento realizado en el software y el uso de la estructura condicional explícita en el enunciado. Durante este proceso P1 permitió a los estudiantes que entre "todos" evaluaran las conjeturas presentadas. Terminada esta actividad, fueron escogidas y reescritas dos. Aquí analizamos una de ellas: Si A y B pertenecen a una circunferencia de centro $C$, entonces $C$ pertenece a la mediatriz del segmento $A B$.

En la cuarta sesión tuvo lugar, en primera instancia, una conversación matemática, y luego se realizó un trabajo en pequeños grupos (trabajo de estudiantes). En dicha conversación matemática P1 y P2 lideraron una actividad enfocada a que "toda la clase" de manera colectiva proporcionara ideas claves para hacer la demostración de la conjetura (esta era la primera vez que una actividad tal se llevaba a cabo en el curso, lo cual la caracteriza como una Tarea Nueva). En ese momento todos los elementos teóricos (definiciones, postulados, teoremas) que intervenían en la demostración hacían parte del sistema teórico disponible. Luego, realizado el trabajo de "toda la clase", cada grupo recibió una hoja con el enunciado de la conjetura, su reformulación y espacio para escribir la demostración en formato a doble columna Afirmación-Garantía (ver Tabla 1). La actividad de los alumnos, organizados en las parejas conformadas desde el principio, se centró entonces en la producción autónoma de un texto escrito de la demostración (actividad que constituye otra Tarea Nueva para la clase). Sobre esta cuarta sesión se elabora el análisis respectivo, que se comunica en este artículo.

Con el propósito de recolectar los datos de la investigación, las sesiones de clase fueron grabadas en audio y video. Se hizo una transcripción completa de la interacción llevada en cada sesión. Asimismo, las producciones escritas de los estudiantes fueron fotocopiadas. Los registros (trascripciones, fotocopias y producciones escritas) se utilizaron para constituir los datos investigativos (que serán descritos en la siguiente sección) y hacer el respectivo análisis, a la luz de los elementos expuestos en la Tabla 2. 


\section{DESCRIPCIÓN Y ANÁLISIS}

Los datos de investigación en los que se centró el análisis fueron dos episodios de la cuarta sesión de clases, donde se abordó el problema antes presentado. El primero de estos episodios tuvo ocho momentos durante los cuales hubo una interacción colectiva, con la que se pretendía la construcción de la demostración de la conjetura (la numeración corresponde a tales momentos):

Producción de la conjetura:

1. Instrucción del profesor respecto a la actividad de clase y repaso de la actividad realizada hasta el momento.

2. Reformulación de la conjetura.

Construcción colectiva de la demostración sobre las siguientes afirmaciones:

3. $\overline{C A}$ y $\overline{C B}$ congruentes.

4. ¿'Y para qué nos sirve esto $(\overline{C A} \cong \overline{C B})$ ?

5. $\triangle C M A$ y $\triangle C M B$ congruentes.

6. $\angle C M A$ y $\angle C M B$ son pares lineales y rectos.

7. $\overleftrightarrow{C M}$ es mediatriz del segmento $\overrightarrow{A B}$.

8. Explicación auxiliar para identificar que la $\overleftrightarrow{C M}$ es mediatriz del segmento $\overline{A B}$.

El segundo episodio corresponde a la escritura autónoma de la demostración por parte de cada grupo de estudiantes (momento 9). Los numerales 3 al 7 corresponden a aserciones estructurales de índole geométrico (Núcleos), sobre los cuales se soporta la demostración de la conjetura. Teniendo en mente este contexto es posible concretar indicadores que dan cuenta de un discurso reflexivo por parte de los alumnos y las normas sociomatemáticas asociadas, ambos elementos presentados en la Tabla 2. En primera instancia se espera que durante la clase en la que se elabora la demostración conjuntamente, los estudiantes actúen en correspondencia con la $\mathrm{NS}_{1}$ (toda afirmación debe argumentarse en el marco del sistema teórico); en ese caso, podría producirse un cambio en su discurso $\left(D_{1}\right)$ por cuanto ellos aludirían, explícitamente, a garantías teóricas para justificar afirmaciones, asunto que hasta ese momento no era usual en su práctica discursiva. Por otro lado, se espera que, en el momento de la escritura autónoma de tal demostración, aludan a tales pasos estructurales de la misma (ver pasos sombreados de la Tabla 1), esto es, a sus Núcleos y Pilares (indicador de cambio de discurso $D_{2}$ ). Se espera, además, que apelen a una estructura de Nivel 2 que contiene todas las Afirmaciones y 
Garantías de la demostración (indicador de cambio de discurso $\mathrm{D}_{3}$ ). Concebimos una correspondencia entre los tipos $\mathrm{D}_{2}$ y $\mathrm{D}_{3}$, con la $\mathrm{NS}_{2}$ (una demostración es una cadena de argumentos válida en matemáticas), pues tales discursos (verbales o escritos) hacen evidente una cadena de argumentos: el primero de pasos estructurales de Nivel 1 o Núcleos-Pilares, el segundo de pasos estructurales y finos o Afirmaciones-Garantías. Para este caso, un discurso reflexivo por parte de los estudiantes puede tener lugar cuando se disponen a escribir autónomamente la demostración puesto que, en ese proceso, deben identificar los argumentos previamente abordados en la construcción colectiva de la demostración, y reflexionar sobre los mismos para, por ejemplo, descartar los que no consideren pertinentes, incluir otros no presentes en la construcción colectiva, organizarlos en una cadena deductiva y presentarlos ordenadamente en el formato que han provisto los profesores P1 y P2 para ello (indicador de discurso reflexivo $\mathrm{D}_{4}$ ).

Por motivos de espacio, en este artículo se presenta la descripción de los momentos 2, 3 y 9, así como el análisis de los momentos 3 y 9. Vale decir que el momento 3 fue el más rico en la interacción del aula, el de mayor duración y el que proporcionó la información más relevante, junto con el momento 9. En las descripciones y análisis se destaca con I y J a los estudiantes que más participaron en la interacción, debido a que nos interesó comparar su actuación durante la interacción colectiva con su producción individual posterior, cuando escriben la demostración. Para sistematizar el análisis usamos los códigos escritos en negrita en la Tabla 2.

\section{MOMENTO 2. REFORMULACIÓN DE LA CONJETURA}

P1 escribe en el tablero "si...entonces..." y dice que, con relación a la conjetura, como ya es usual, se deben precisar dos "cosas" importantes: su hipótesis y tesis. Da tiempo para que los alumnos digan a qué se hace referencia cuando se habla de hipótesis en una demostración. Un estudiante expresa que a lo "dado". P1 pregunta qué sería lo "dado" en el enunciado de la conjetura, y "aquello a lo que se quiere llegar". Otro estudiante (E2) responde las preguntas, a la vez que P1 escribe lo "dado" frente a la palabra "hipótesis" y "aquello a lo que se quiere llegar" frente de la palabra "tesis" (Figura 1). P1 reescribe, con notación geométrica, tanto la hipótesis como la tesis. Finalmente, en el tablero queda escrito lo siguiente: 

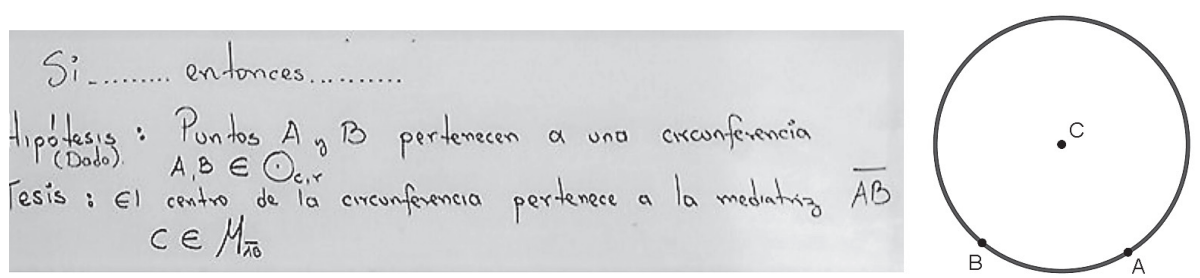

Figura 1. Hipótesis y tesis de la conjetura.

\section{MOMENTO 3. CONSTRUCCIÓN COLECTIVA DE LA DEMOSTRACIÓN: SEGMENTOS CA Y CB CONGRUENTES}

Dada la extensión de este tercer momento, y con el ánimo de facilitar la interpretación del análisis elaborado, al mismo tiempo que se describe la interacción, se irá colocando entre corchetes cada uno de los códigos del dispositivo analítico. Cuando se considere pertinente, se hará una síntesis del mismo. Para iniciar la Tarea Nueva (i.e., la construcción colectiva de la demostración), P1 solicita a una de las estudiantes (E3) del curso que, con palabras, diga qué es lo que se tiene que demostrar $\left[\mathrm{CDP}_{1}\right]$. E3 responde: "que $\mathrm{C}$ pertenece a la mediatriz del segmento AB". P1 avala la respuesta. Pregunta a todos los alumnos: ¿Esto es lo dado para nosotros?, señalando la hipótesis expuesta en la Figura 1 [CDP $]$. Un estudiante (J) responde que sí, y ante la pregunta ¿por qué?, J da una respuesta avalada por el profesor: "porque cumple con las condiciones de la hipótesis, que los puntos $\mathrm{A}$ y $\mathrm{B}$ pertenecen a la circunferencia" $\left[\mathrm{CDE}_{3}, \mathrm{CDE}_{4}\right]$. La interacción correspondiente se presenta en la Tabla 3. 
Diferencias entre discursos colectivos (verbales) e individuales (escritos)...

Tabla 3. Interacción Construcción colectiva de la demostración: Segmentos CA y CB congruentes.

\begin{tabular}{|c|c|c|c|}
\hline \multicolumn{3}{|r|}{ Interacción } & \multirow{2}{*}{$\begin{array}{l}\text { Análisis } \\
{\left[\mathrm{CDP}_{1}\right] \text { Favorece participación de estudiantes }} \\
\text { haciendo una pregunta que pretende involucrarlos } \\
\text { en un discurso argumentativo. }\end{array}$} \\
\hline 1 & $\mathrm{P} 1$ & $\begin{array}{l}\text { ¿Yo puedo tener estos dos } \\
\text { segmentos }(\overline{A C}, \overline{B C}) \\
\text { congruentes? [Señala radios } \\
\text { de la circunferencia } \\
\text { representada en la Figura } 1]\end{array}$ & \\
\hline 2 & $\mathrm{~V}^{17}$ & Sí. & $\begin{array}{l}{\left[\mathrm{CDE}_{4}\right] \text { Participan en clase de una manera simplista, }} \\
\text { siguiendo el contrato didáctico (de la situación } \\
\text { instruccional tradicional): responden las preguntas } \\
\text { del profesor. }\end{array}$ \\
\hline 3 & $\mathrm{P} 1$ & ¿Por qué? & $\begin{array}{l}\left.\text { [CDP }{ }_{1}\right] \text { Pregunta centrada en la búsqueda de } \\
\text { argumentos para la construcción de la demostración. } \\
\text { [TN] P1 intenta generar un ambiente en el cual se } \\
\text { deba justificar las afirmaciones en el marco de una } \\
\text { demostración. Está en la búsqueda de la construcción } \\
\text { de } \mathrm{NS}_{1} \text { (toda afirmación debe argumentarse con } \\
\text { elementos del sistema teórico de la clase). }\end{array}$ \\
\hline 4 & I & $\begin{array}{l}\text { C es el centro de la } \\
\text { circunferencia, y A y B son } \\
\text { dos puntos que pertenecen a } \\
\text { ella, pues sería que el } \\
\text { segmento AC y el segmento } \\
\text { BC serían radios, son dos } \\
\text { radios de la circunferencia, y } \\
\text { entonces son congruentes. }\end{array}$ & $\begin{array}{l}{\left[\mathrm{CDE}_{3}, \mathrm{CDE}_{4}\right] \text { Participa en la clase autónomamente, }} \\
\text { pero además intenta dar explicaciones (palabras en } \\
\text { negrilla). No hay alusión explícita a garantías } \\
\text { contenidas en el sistema teórico, pero sí una } \\
\text { implícita (en cursiva), que tiene que ver con el } \\
\text { hecho: dos radios de una circunferencia son } \\
\text { congruentes. Está en busca de la NS1. }\end{array}$ \\
\hline 5 & $\mathrm{P} 1$ & $\begin{array}{l}\text { [Escribe en el tablero } \\
\overline{A C} \cong \overline{B C} \text { Ese (se refiere a lo } \\
\text { dicho por I) sería un } \\
\text { argumento para decir que } \\
\text { son congruentes. Les } \\
\text { pregunto a todos, ¿̇erá el } \\
\text { único? ¿Será que hay más } \\
\text { argumentos? }\end{array}$ & $\begin{array}{l}\left.\text { [CDP }_{1}, \mathrm{TN}\right] \text { Pese a que I provee un argumento, P1 } \\
\text { sigue indagando en busca de otros argumentos, } \\
\text { bien sea para ajustar, complementar o corregir el ya } \\
\text { provisto, o sencillamente proveer otros, para que } \\
\text { "toda la clase" tenga ocasión de escoger el mejor. } \\
\text { Todavía no se instaura NS1, pues no gestiona la } \\
\text { explicitación de la garantía. }\end{array}$ \\
\hline 6 & V & No. & $\begin{array}{l}{\left[\mathrm{CDE}_{4}\right] \text { Participan en clase de una manera simplista, }} \\
\text { respondiendo sí o no. }\end{array}$ \\
\hline
\end{tabular}

${ }^{17} \vee$ significa Varios estudiantes. 


\begin{tabular}{|c|c|c|c|}
\hline 7 & P1 & ¿Por qué no? & $\begin{array}{l}{\left[\mathrm{CDP}_{1}, \mathrm{TN}\right] \text { Sigue indagando en busca de otros }} \\
\text { argumentos. }\end{array}$ \\
\hline 8 & J & $\begin{array}{l}\text { Porque son los únicos puntos } \\
\text { [se refiere a A y B] que están } \\
\text { a la misma distancia al } \\
\text { centro. }\end{array}$ & $\begin{array}{l}{\left[\mathrm{CDE}_{4}\right] \text { Participa en la clase autónomamente, pero }} \\
\text { además intenta dar explicaciones (aludiendo a la } \\
\text { palabra en negrilla). Sin embargo, no hay alusión } \\
\text { explícita a garantías del sistema. Todavía no se } \\
\text { instaura } \mathrm{NS}_{1} \text {. }\end{array}$ \\
\hline 9 & P1 & $\begin{array}{l}\text { I dice que los dos segmentos } \\
\text { son congruentes porque son } \\
\text { dos radios de una } \\
\text { circunferencia, o sea, está } \\
\text { teniendo en cuenta la } \\
\text { definición de radio, ¿cierto? }\end{array}$ & $\begin{array}{l}\left.\mathrm{CDP}_{3}, \mathrm{CDP}_{2}\right] \text { El profesor parafrasea lo dicho por l; } \\
\text { provee una garantía (en negrilla) asociada a lo } \\
\text { enunciado por I. No obstante, tal garantía no es } \\
\text { correcta, tal definición no alude a congruencia. }{ }^{18} \\
\text { Todavía no se instaura } \mathrm{NS}_{1} \text {, pero P1 actúa en } \\
\text { conformidad con ella. Puede estar mostrando un } \\
\text { modelo de acción para que los estudiantes lo sigan. }\end{array}$ \\
\hline 10 & V & Asienten con la cabeza. & $\begin{array}{l}{\left[\mathrm{CDE}_{4}\right] \text { Participan en clase de una manera simplista, }} \\
\text { respondiendo sí o no, como consecuencia del } \\
\text { contrato didáctico usual (de la situación } \\
\text { instruccional tradicional). }\end{array}$ \\
\hline 11 & P1 & $\begin{array}{l}\text { ¿Será la única forma para } \\
\text { determinar que estos dos } \\
\text { segmentos son congruentes? }\end{array}$ & $\begin{array}{l}{\left[\mathrm{CDP}_{1}, \mathrm{TN}\right] \text { Sigue indagando en búsqueda de otros }} \\
\text { argumentos. Todavía no se instaura } \mathrm{NS}_{1} \text {. }\end{array}$ \\
\hline 12 & J & $\begin{array}{l}\text { También podría decir por } \\
\text { definición de equidistancia. }\end{array}$ & $\begin{array}{l}{\left[\mathrm{CDE}_{3}, \mathrm{CDE}_{4}\right] \text { Participa en la clase autónomamente, }} \\
\text { usando lenguaje geométrico. } \\
{\left[\mathrm{NS}_{1}, \mathrm{CDE}_{1}\right] \text { Provee una garantía teórica, resaltada }} \\
\text { en negrilla. Actúa en consonancia con la } \mathrm{NS}_{1} \text {. }\end{array}$ \\
\hline 13 & P1 & $\begin{array}{l}\text { Ah, por definición de } \\
\text { equidistancia. J recuérdanos } \\
\text { la definición de equidistancia. }\end{array}$ & $\begin{array}{l}{\left[\mathrm{CDP}_{1}\right] \text { incentiva la participación de J, favoreciendo }} \\
\text { su participación. }\end{array}$ \\
\hline 14 & J & $\begin{array}{l}\text { [leyendo su cuaderno] Los } \\
\text { puntos } \mathrm{D} \text { y B equidistan de } \mathrm{C} \\
\text { si } \mathrm{DC}=\mathrm{BC} \text {. Entonces se puede } \\
\text { decir que los puntos } \mathrm{A} \text { y } \mathrm{B} \\
\text { equidistan de } \mathrm{C} \text {, si } \mathrm{AC}=\mathrm{BC} \text {. }\end{array}$ & $\begin{array}{l}{\left[\mathrm{CDE}_{3}, \mathrm{CDE}_{4}\right] \text { Da respuesta a la solicitud del profesor; }} \\
\text { además provee un ejemplo de la Definición de } \\
\text { Equidistancia, particularizado a la situación. Ratifica } \\
\text { su actuación en consonancia con la } \mathrm{NS}_{1} \text {. }\end{array}$ \\
\hline
\end{tabular}

18 Definición de radio: Dada una circunferencia de centro $C$. El segmento $A C$ es radio de tal circunferencia si y solo si $A$ pertenece a dicha circunferencia. 
Simultáneamente, I y P2 reaccionan ante lo dicho por J:

15 I Pero tocaría tener en cuenta la $\left[\mathrm{CDE}_{3}, \mathrm{CDE}_{4}\right]$ Participa autónomamente en la definición de circunferencia. actividad de la clase, asumiendo una posición complementaria respecto a lo dicho por J. $\left[\mathrm{NS}_{1}, \mathrm{CDE}_{1}\right]$ Provee una garantía teórica, resaltada en negrilla. Actúa en consonancia con la NS1.

16 P2 Pero ccómo sabes tú $\quad\left[C_{1} P_{1}\right]$ P2 indaga a I respecto a su pregunta. [dirigiéndose a J] que esos $\quad\left[\mathrm{CDP}_{2}\right] \mathrm{P} 2$ sabe que debe seguir apoyando la puntos equidistan? participación de J, pues él está en una vía adecuada para justificar la congruencia en cuestión.

17 I Ahí es donde hay que tener $\left[\mathrm{NS}_{3}, \mathrm{NS}_{4}, \mathrm{CDE}_{3}, \mathrm{CDE}_{4}\right]$ Participa autónomamente en en cuenta la definición de circunferencia. la actividad de la clase, asumiendo una posición complementaria respecto a lo dicho por J.

$\left[\mathrm{NS}_{1}, \mathrm{CDE}_{1}\right]$ Provee una garantía teórica, resaltada en negrilla. Actúa en consonancia con la $\mathrm{NS}_{1}$.

18 P2 Y ¿qué dice la definición de circunferencia? [Dirigiéndose a l]

$\left[\mathrm{CDP}_{1}\right]$ Incentiva la participación de I, favoreciendo su participación.

19 I [leyendo su cuaderno] Son los $\left[\mathrm{CDE}_{3}, \mathrm{CDE}_{4}\right]$ Da respuesta a la solicitud del profesor. puntos de un plano que equidistan de un punto dado del mismo plano.

Como se puede observar, la forma de intercambio (Jones \& Herbst, 2012) que tiene lugar en ese momento de la clase es una Tarea Nueva (TN), esto es, P1 propone, quizá implícitamente, la elaboración conjunta de la demostración de una conjetura. En especial, el asunto se centra en la construcción de uno de los pasos estructurales de argumentación de tal demostración $(\overline{A C} \cong \overline{B C}$ ). En la interacción destacan cuatro elementos que guardan relación con el dispositivo analítico:

1. Los estudiantes que participan en la interacción tienen un discurso que corresponde principalmente con las $\mathrm{NS}_{3}$, usan un lenguaje geométrico que ha sido instaurado en las clases previas y procuran que su participación aporte a la actividad matemática (v.g., responden a las preguntas del profesor usando terminología geométrica estudiada en el curso). 
2. La interacción muestra un ejemplo de la manera como se construye (o negocia) la $\mathrm{NS}_{1}$ (Yackel \& Cobb, 1996). P1, I y J intentan proveer garantías para argumentar sus afirmaciones. En un primer momento (líneas 1-9) los alumnos exponen garantías que no son parte del sistema teórico de la clase; es el maestro quien en la línea 9 provee una que sí está contenida en dicho sistema. Luego (líneas 9-19) los estudiantes parecen interpretar el mensaje del docente y, de manera autónoma, empiezan a dar garantías que sí son parte del sistema (aluden a las definiciones de equidistancia y de circunferencia) durante la interacción que tiene por objeto complementar la idea de J. Como resultado de la interacción y negociación producida en este momento, tiene lugar un primer indicio del cambio de discurso en los alumnos (I y J), hacia $\mathrm{D}_{1}$, puesto que usan explícitamente garantías teóricas para justificar afirmaciones. Como no se han discutido más pasos de la demostración, no ha habido ocasión para aludir a la $\mathrm{NS}_{2}$.

3. Solo dos alumnos $(\mathrm{I}, \mathrm{J})$ cumplen reiteradas veces con cláusulas del contrato $\left(\mathrm{CDE}_{1}, \mathrm{CDE}_{3}, \mathrm{CDE}_{4}\right)$, hecho que explica en gran medida por qué tiene lugar una negociación de la $\mathrm{NS}_{1}$ que lo involucra, y del consecuente cambio de discurso (hacia $\mathrm{D}_{1}$ ) por parte de ellos.

4. En correspondencia con TN, la cláusula del contrato didáctico más frecuente por parte del profesor (y por ende, que describen su rol) es $\mathrm{CDP}_{1}$ : posibilita la participación de los estudiantes preguntándoles reiteradamente el porqué de ciertas afirmaciones. Esto concreta la intención de P1 para generar aprendizaje en los alumnos (producir argumentos válidos en geometría $-\mathrm{NS}_{1}-$ ), motivando la constitución de una cultura de indagación en el aula.

Enseguida presentamos la continuación de la interacción (Tabla 4), con su respectivo análisis. P2 toma la batuta de la clase, hace un recuento desde el momento en que J participa en la interacción, e involucra a los estudiantes en el mismo: 
Tabla 4. Continuación interacción Construcción colectiva de la demostración: Segmentos CA y CB congruentes.

\begin{tabular}{|c|c|c|c|}
\hline & & Interacción & nálisis \\
\hline 20 & P2 & $\begin{array}{l}\text { ¿Qué tenemos primero? Que los } \\
\text { puntos pertenecen a la } \\
\text { circunferencia, ¿cierto? }\end{array}$ & \multirow{7}{*}{$\begin{array}{l}\text { Producto del análisis de las argumentaciones } \\
\text { hechas hasta el momento }\left[\mathrm{CDP}_{2}\right], \text { P2 recapitula lo } \\
\left.\text { hecho con el fin encaminar la discusión } \mathrm{CDDP}_{3}\right] \\
\text { Para este caso, precisar lo que permite garantizar } \\
\text { la congruencia en cuestión. Indaga sobre la } \\
\text { validez de incluir "radios" en la demostración. } \\
{\left[\mathrm{CDE}_{3} \text {; } \mathrm{CDE}_{4}\right] \text { I y J responden las preguntas de }} \\
\text { P2. Probablemente J dice "radio", pues P1 aludió } \\
\text { a este objeto en una intervención anterior y es } \\
\text { parte del contrato de la clase tradicional } \\
\text { aceptar lo dicho por el profesor. Otra } \\
\text { interpretación posible es que algunos } \\
\text { estudiantes estarían acostumbrados a } \\
\text { involucrar radios siempre que hacen referencia } \\
\text { a una circunferencia, aun cuando tal objeto } \\
\text { (radios) no está presente (ni es necesario) en la } \\
\text { definición de circunferencia. }\end{array}$} \\
\hline 21 & J, I & $\begin{array}{l}\text { [Solo ellos asienten con la } \\
\text { cabeza] }\end{array}$ & \\
\hline 22 & $\mathrm{P} 2$ & Como pertenecen ¿qué pasa? & \\
\hline 23 & I & Son equidistantes. & \\
\hline 24 & J & Son equidistantes de $\mathrm{C}$. & \\
\hline 25 & P2 & $\begin{array}{l}\text { Al tener equidistancia, ¿̇ué } \\
\text { tenemos? }\end{array}$ & \\
\hline 26 & J & Los radios. & \\
\hline 27 & $\mathrm{P} 2$ & $\begin{array}{l}\text { ¿Ahí tenemos que meter los } \\
\text { radios? }\end{array}$ & \multirow{3}{*}{$\begin{array}{l}{\left[\mathrm{CDP}_{2}, \mathrm{CDP}_{3}\right] \text { P2 aclara por qué no es necesario }} \\
\text { aludir a "radio" en el argumento solicitado por } \\
\text { P1, por cuanto menciona que con la } \\
\text { equidistancia se tiene información suficiente } \\
\text { para aludir a la congruencia en cuestión. Igual } \\
\text { que antes, probablemente algunos estudiantes } \\
\text { están acostumbrados a involucrar radios } \\
\text { siempre que hacen referencia a una } \\
\text { circunferencia. } \\
\left.\text { [CDP }{ }_{1}\right] \text { P2 pregunta por una garantía que } \\
\text { justifique dicha equidistancia. }\end{array}$} \\
\hline 28 & I & No. & \\
\hline 29 & P2 & $\begin{array}{l}\text { Pues esa sería una información } \\
\text { como adicional, ¿no? [Enuncia la } \\
\text { definición de radio]. }{ }^{19} \text { Pero tenerlo } \\
\text { no es una información relevante, } \\
\text { puesto que ya se tiene la } \\
\text { equidistancia y como lo que } \\
\text { preguntaba el profesor P1 era } \\
\text { "por qué esos dos segmentos [AC } \\
\text { y BC] eran congruentes... listo". O } \\
\text { sea, lo tenemos ¿ipor qué cosa? }\end{array}$ & \\
\hline
\end{tabular}

${ }^{19}$ Definición de Radio: Un radio de una circunferencia es el segmento cuyos extremos son el centro y un punto de la misma 


\begin{tabular}{|c|c|c|c|}
\hline 30 & E4 & Por definición de equidistancia. & $\begin{array}{l}\left.\mathrm{NS}_{1}, \mathrm{CDE}_{3}, \mathrm{CDE}_{4}\right] \mathrm{E} 5 \text { parece entender que se } \\
\text { pregunta por la congruencia, no por la } \\
\text { equidistancia. Actúa en correspondencia con } \\
\mathrm{NS}_{1} \text {, pues sugiere una garantía teórica. }\end{array}$ \\
\hline 31 & P2 & Por definición de... & $\begin{array}{l}{\left[\mathrm{CDP}_{1}\right] \text { Reitera la pregunta, con el fin de corregir }} \\
\text { lo dicho por E4. }\end{array}$ \\
\hline 32 & V & Por definición de circunferencia. & $\begin{array}{l}{\left[\mathrm{NS}_{1}, \mathrm{CDE}_{3}, \mathrm{CDE}_{4}\right] \text { Proveen una garantía }} \\
\text { solicitada. }\end{array}$ \\
\hline 33 & P2 & $\begin{array}{l}\text { Y como son equidistantes ¿̇quiere } \\
\text { decir que qué? }\end{array}$ & $\begin{array}{l}{\left[\mathrm{CDP}_{1}\right] \text { Indaga, esta vez por la conclusión }} \\
\text { seguida luego de usar la garantía "definición } \\
\text { de equidistancia". }\end{array}$ \\
\hline 34 & J & $A C=B C$ & $\begin{array}{l}{\left[\mathrm{CDE}_{3}, \mathrm{CDE}_{4}\right] \text { Responde adecuadamente, }} \\
\text { proveyendo una conclusión válida. }\end{array}$ \\
\hline 35 & P2 & $\begin{array}{l}\text { Tienen la misma medida, } \\
\text { ¿Entonces? }\end{array}$ & {$\left[\mathrm{CDP}_{1}\right]$ Parafrasea lo dicho por J. } \\
\hline 36 & E5 & Son congruentes. & $\begin{array}{l}{\left[\mathrm{CDE}_{3}, \mathrm{CDE}_{4}\right] \text { Responde adecuadamente, }} \\
\text { proveyendo una conclusión válida. Terminado } \\
\text { el episodio, se tiene evidencia de una actuación } \\
\text { en correspondencia con la NS2, pues hay una } \\
\text { producción de una cadena deductiva. }\end{array}$ \\
\hline
\end{tabular}

Comentamos dos aspectos sobre la interacción anterior:

1. P2 toma el rol correspondiente a la cláusula $\mathrm{CDP}_{3}$ (líneas 20-29). Luego de hacer un análisis de los argumentos producidos hasta el momento [CDP ${ }_{2}$, P2 precisa los argumentos que posibilitan $\overline{A C} \cong \overline{B C}$, y reorienta la interacción llevada a cabo para que ello sea posible $\left[\mathrm{CDP}_{3}\right]$. Descarta la alusión a "radio" realizada por P1, hecho que se esperaba, pues su labor en el aula era apoyar a tal profesor en el momento oportuno. Con esta acción P2 intenta hacer claro, para los estudiantes que involucraron radios, por qué es suficiente usar la definición de circunferencia y de equidistancia como garantías, sin necesidad de aludir a dicho objeto recordando su definición.

2. Pareciera que se ha instaurado la $\mathrm{NS}_{1}$. Otros alumnos $(30,32)$ participan en la interacción proveyendo garantías que son parte del sistema teórico. Por otro lado, es la primera vez que se actúa en conformidad a $\mathrm{NS}_{2}$ : se genera una primera cadena de argumentos producto de la interacción (que alude 
a pasos de nivel 2, resaltados en negrilla en la Tabla 5). ${ }^{20}$ Sin embargo, no es posible decir aún si esta se convierte en norma sociomatemática de la clase, puesto que los estudiantes no generan cadenas deductivas autónomamente. Como consecuencia de lo anterior, ellos muestran evidencia de un discurso $D_{1}$, pero todavía no hay evidencia de un discurso $D_{2} \circ D_{3}$. Los profesores pretendían que los estudiantes produjeran tales discursos más adelante, específicamente durante la construcción colectiva relativa al momento 6, por ejemplo, y en la escritura autónoma de la demostración. ${ }^{21}$

Tabla 5. Primera cadena de argumentos.

\begin{tabular}{c|l|l}
\hline 1. & Puntos $A$ y $B$ & Dado \\
\hline 2. & $A, B \in \odot_{C, r}$ & Dado \\
\hline 3. & $A, B$ equidistan de $C A$ & D. Circunferencia (2) \\
\hline 4. & $A C=B C$ & Def. equidistancia (3) \\
\hline 5. & $\overline{A C} \cong \overline{B C}$ & Def. congruencia de segmentos (4) \\
\hline
\end{tabular}

\section{MOMENTO 9. ESCRITURA AUTÓNOMA DE LA DEMOSTRACIÓN}

En este momento los alumnos, por parejas y de manera autónoma, escriben la demostración de la conjetura, aludiendo a todos los pasos discutidos en la actividad colectiva. Para ello, P1 da una instrucción según la cual cada pareja debe escribir todos los pasos de la demostración, entrega una hoja que presenta el enunciado de la misma, su reformulación explicitando hipótesis y datos, y una tabla a tres columnas (una para poner el numeral del paso, otra para las Afirmaciones y otra para la Garantía. Ver Figura 2). Pese a que esta es la primera vez que se hace una actividad en la que se produce una conjetura y se construye colectivamente su demostración, los estudiantes están familiarizados con el formato porque P1 lo utilizaba en la situación instruccional tradicional de la clase, consistente en exponer la demostración de un hecho. Vale

\footnotetext{
${ }^{20}$ Vale decir que estos pasos son, en esencia, equivalentes a los pasos 1 al 6 presentados en la Tabla 1.

21 En efecto, durante el momento 6, donde se pretendía inferir que y son pares lineales y rectos, se hizo una construcción colectiva donde se presentaron pasos de nivel 2 con cierto detalle. De nuevo I fue el estudiante más activo en dicha interacción. En él se aludió a los pasos 14 al 20 de la Tabla 1.
} 
decir que durante la TN de construir colectivamente la demostración, no hubo escritura ordenada en el tablero de los pasos de la demostración en cuestión; eventualmente P1 escribió algunas ideas para facilitar la comunicación. Nuestro análisis se centra en la producción del equipo de I (no se tiene en cuenta a J por déficit de espacio), el estudiante más activo en toda la construcción colectiva de la demostración y que tuvo una producción discursiva $\mathrm{D}_{1}$ (provee garantías teóricas a afirmaciones) que se puede avalar como correcta. Se pretende precisar si l experimentó una reflexión sobre el discurso para poder sistematizar la demostración $\left(D_{4}\right)$, teniendo como base, a su vez, otros tipos de discurso $\left(D_{2}\right.$ : escritura de pasos estructurales -Núcleos y Pilares- de Nivel 1 de la demostración; $D_{3}$ : escritura de pasos de Nivel 2 de la demostración).

\begin{tabular}{|c|c|c|}
\hline No. & Afirmación & Garantía \\
\hline 1 & $A, B \in \bigodot_{c, r}$ & - Dado \\
\hline 2 & Trazamos $\overline{A B}$ & Construccion Auxiliar [1]. \\
\hline 3 & T. $M$ punto medio de $\overline{A B}$ & Construccion Axithar [2] \\
\hline $4^{*}$ & $\overline{B C}$ y $\quad \overline{A C}$ & Constrvcaín Auxiliar [1]. \\
\hline 5. & $\overline{A C} \cong$ & Definicion de circunferencia [1] \\
\hline
\end{tabular}

Figura 2. Parte del discurso escrito de la demostración del equipo de I.

Análisis. I tiene un rol principal en la escritura de la demostración, su compañero actúa de manera secundaria en la actividad. En consecuencia, no es inapropiado pensar que la producción escrita de I alude a su propio discurso. Enseguida, se presenta el análisis en términos de los discursos $D_{1}$, $\mathrm{D}_{2}, \mathrm{D}_{3}$ y $\mathrm{D}_{4}$

En relación con $D_{1}$ y $D_{2}$, la demostración escrita alude a todos los pasos estructurales de Nivel 1 de la demostración de la conjetura (ver pasos sombreados en la Tabla 1 ) para el caso en que $C$ no pertenece a $\overline{A B}$ (en clase no se abordó el caso contrario); los Núcleos y Pilares correspondientes a estos pasos fueron válidos conceptual $\left(\mathrm{NS}_{1}\right)$ y escrituralmente $\left(\mathrm{NS}_{3}\right)$. Además, fueron puestos en el orden correcto, generando una cadena deductiva válida (en correspondencia $\mathrm{NS}_{2}$ ), lo cual es un indicador de que I toma los argumentos construidos en la actividad de toda la clase y es capaz de plasmarlos en el 
formato provisto por el maestro para comunicar su demostración (tuvo un discurso reflexivo $\left.\mathrm{D}_{4}\right)^{22}$

Un aspecto relevante es que, si bien esta fue una actividad autónoma por parte de los estudiantes, hay una acción del profesor, quien está implícitamente presente e induce al uso de los discursos $D_{1}$ y $D_{2}$ (no necesariamente válidos): el formato para elaborar la demostración alude a las columnas Afirmación y Garantía (relativa a $D_{1}$ ), y a la necesidad de hacer varios pasos encadenados (relativo a $\mathrm{D}_{2}$ ) producto de la aparición de varias filas. Esta acción del docente está en el terreno de una forma de intercambio de tipo situación instruccional (SI), puesto que se reitera implícitamente la cláusula $\mathrm{CDP}_{1}$ y la necesidad de cumplir con $\mathrm{NS}_{1}$ y $\mathrm{NS}_{2}$.

Respecto a $\mathrm{D}_{3}$ (precisión de pasos estructurales de nivel 2), por asuntos de espacio solo se alude a la primera parte de la demostración escrita, es decir, hasta el paso discutido en el momento 3 (Figura 2). Al hacer una comparación con la Tabla 1, vemos que hay diferencias fundamentales en los pasos previos a la inferencia de $\overline{A C} \cong \overline{B C}$ : en la producción de I no existe alguna alusión a los pasos "finos" que permitieron deducir tal congruencia (pasos en negrilla de la Tabla 5), pero sí a unos de construcción (P1 ha instruido en relación con ello). En definitiva, no hay un cambio en el discurso de tipo $D_{3}$, aun cuando I tuvo una participación relevante en la interacción donde tuvo lugar el estudio respectivo (momento 3). ¿Qué pudo ocurrir si, en ese momento, el estudiante actuó en conformidad con $N S_{1}$ y las respectivas cláusulas del contrato $\left(C D E_{1}, C D E_{3}\right.$, $\left.C D E_{4}\right)$, y $P 1$ actuó en correspondencia con $C D P_{1}, y C D P_{3}$ ?

Como respuesta se podría pensar que, en ese momento, $\mathrm{NS}_{2}$ no se había constituido como norma de la clase; más bien, se considera que tal norma fue constituida parcialmente. Dicha norma se intentó constituir de manera similar a como ocurrió con $\mathrm{NS}_{1}$ (no de manera explícita, sino como producto de la interacción mediada por las cláusulas de contrato didáctico); sin embargo, aunque la demostración escrita (o discurso) de I contiene una cadena deductiva con todos los pasos estructurales -Núcleos y Pilares- $\left(D_{2}\right)$, tal discurso no presenta los pasos "finos" o estructurales de Nivel 2 que sustenta $\overline{A C} \cong \overline{B C}$ (no hay

22 Un discurso tipo $D_{2}$ completamente válido (i.e., una cadena deductiva válida que contiene los pasos estructurales en los cuales cada afirmación tiene su garantía válida), implica un discurso tipo $\mathrm{D}_{1}$ válido. La implicación recíproca no necesariamente es verdadera; se puede tener una garantía válida para una afirmación $\left(D_{1}\right)$, pero construir una cadena deductiva $\left(D_{2}\right)$ inválida que la contenga. A su vez, se puede dar un discurso tipo $\mathrm{D}_{2}$ parcialmente válido (i.e., una cadena deductiva válida que contiene los pasos estructurales en los cuales alguna afirmación tiene su garantía inválida). 
$\mathrm{D}_{3}$ ). Así las cosas, I todavía no actúa por completo en conformidad con dicha norma. Es probable que el esfuerzo llevado a cabo por P2 no haya surtido efecto; la exigencia de solicitar las afirmaciones y garantías expuesta en las Tablas 3 y $4\left(\mathrm{CDP}_{1}\right.$ y $\left.\mathrm{CDP}_{3}\right)$ no fue suficiente para que, desde un punto de vista matemático, estos pasos "finos" fueran significativos para I. ${ }^{23}$ Parece pretencioso pensar que es suficiente abordar el tratamiento de pasos "finos" un par de veces y aspirar a una escritura "completa" de una demostración. Posiblemente, hizo falta que el profesor involucrara a los estudiantes en más tareas que favorecieran la explicitación de tales pasos empleando la cláusula $\mathrm{CDP}_{3}$ y que los alumnos asumieran $\mathrm{CDE}_{2}$ con mayor autonomía. Un ambiente tal hubiese favorecido tanto la constitución de $\mathrm{NS}_{2}$ como una reflexión que los concientizara de la necesidad de explicitar dichos pasos $\left(\mathrm{D}_{3}\right)$. Desde esta perspectiva, es razonable pensar que un objeto de conocimiento a largo plazo (Jones \& Herbst, 2012), en este nivel educativo, es la explicitación de los pasos estructurales de Nivel 2 en una demostración; en contraste $\mathrm{D}_{1}$ (proveer garantías teóricas) o $\mathrm{D}_{2}$ (proveer Núcleos-Pilares) es un conocimiento generado en actividades de momento a momento. Pese a lo anterior, de ninguna manera se considera desafortunado que este grupo de estudiantes solo haya provisto pasos estructurales tipo Núcleos y Pilares (de Nivel 1), puesto que ello muestra una comprensión general de la demostración de la conjetura; en otras palabras, los alumnos son capaces de precisar aquellos asuntos modulares que la componen. Vale destacar que una explicitación de los pasos finos de Nivel 2 mostraría con mayor fuerza una argumentación deductiva por parte de los estudiantes, ya que presentarían una sistematización conformada por "todos" los pasos argumentales (cadenas deductivas necesarias) que permiten inferir cada paso modular que compone la demostración (Mejia-Ramos, Fuller, Weber, Rhoads \& Samkoff, 2012).

\section{REFLEXIONES FINALES}

El dispositivo analítico, para el caso del curso de secundaria, permitió tener evidencias para identificar que, en el marco de la Tarea Nueva Construir colectivamente una demostración, el uso continuo (y cíclico) de cláusulas del contrato didáctico por parte del profesor (e.g., hacer preguntas de indagación - $\mathrm{CDP}_{1}^{-}$, y orientar y redireccionar

23 De hecho, no fueron significativos para ningún estudiante (en sus discursos escritos no hay evidencia de tales pasos). 
las producciones de los alumnos $-\mathrm{CDP}_{3}-$-), junto con las respectivas respuestas por parte de los estudiantes en cumplimiento de su parte contractual (e.g., argumentar afirmaciones $-\mathrm{CDE}_{1}-$, y participar en clase $-\mathrm{CDE}_{2}$ y $\mathrm{CDE}_{3}-$ ), llevan a instaurar normas sociomatemáticas como toda afirmación se argumenta con base en el sistema teórico $\left(\mathrm{NS}_{1}\right)$ y una demostración es una cadena deductiva $\left(\mathrm{NS}_{2}\right)$.

Como consecuencia, los estudiantes tienden a producir ciertos tipos de discursos, unos en los que se explicita la garantía teórica de una afirmación $\left(D_{1}\right)$ en una interacción verbal, y otros en los que se explicitan pasos estructurales de Nivel 1 (Núcleos-Pilares) en una demostración escrita $\left(\mathrm{D}_{2}\right)$. Vale decir que, al momento de escribir la demostración, no hubo un discurso de tipo $\mathrm{D}_{3}$ (explicitación de los pasos de Nivel 2 de la demostración); en la sección Momento 9. Escritura autónoma de la demostración, se dieron posibles explicaciones al respecto. En este sentido, la experiencia analítica (para el caso de I), aporta evidencia para decir que en la medida que se asuman reiteradamente las cláusulas por parte del estudiante y haya conciencia de las normas asociadas, existe mayor posibilidad de un cambio en su discurso (verbal y escrito), de forma tal que se corresponda con una demostración válida asociada al Nivel 1 de estructuración. A la luz de lo anterior, es posible sugerir una vía de dirección temporal que relacione cláusulas del contrato, normas sociomatemáticas y tipos de discurso (ver Figura 3).

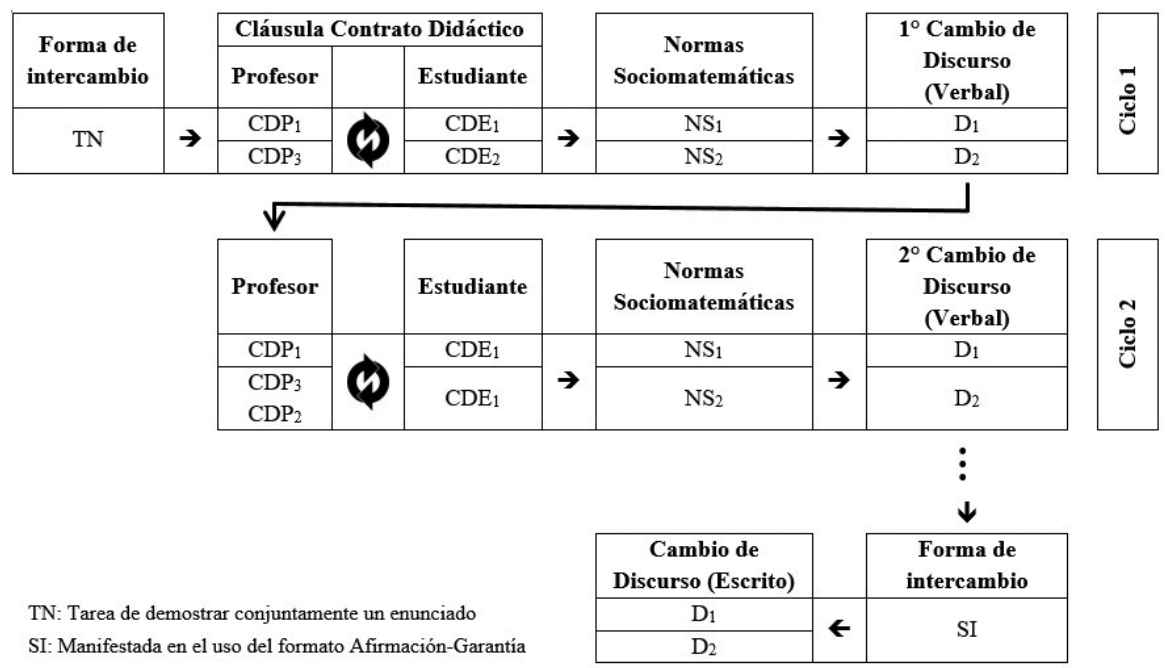

Figura 3. Relación temporal entre formas de intercambio, contrato didáctico, normas sociomatemáticas y cambio de discurso. 
Esta ruta temporal sugiere una manera de actuar en el aula que puede ser exitosa, como se verificó en el análisis (en términos de $D_{1}$ y $D_{2}$ ) para aquellos estudiantes que, junto con los profesores, participan reiteradamente en ciclos de interacción (por eso el símbolo de flecha circulares) cumpliendo las respectivas cláusulas del contrato $\left(\mathrm{CDP}_{1}, \mathrm{CDP}_{3}, \mathrm{CDE}_{1}, \mathrm{CDE}_{2}\right)$. En otras palabras, estos hechos son resultado del sistema de normas contracto didáctico - normas sociomatemáticas (Godino, Font, Wilhelmi \& Castro, 2009) instaurado o negociado en el aula de clase. El sistema surgió a partir de situaciones novedosas para los alumnos al instaurar nuevas normas que se constituyen en (o pretenden conducir a) nuevos aprendizajes (Jones \& Herbst, 2012) que se evidencian en sus cambios (o no) de discurso o en sus discursos reflexivos (Cobb, Boufi, McClain \& Whitenack, 1997).

Por supuesto, la ruta temporal ilustrada en la Figura 3 no muestra una verdad absoluta, solo pretende proveer, por un lado, una posible ruta de interacción que favorece una modificación del discurso de los estudiantes que participan en ella, y por otro, una manera alternativa para instaurar normas sociomatemáticas. Usualmente, se cree que estas normas se deben explicitar al inicio de una clase por parte del profesor; el análisis mostró que para este caso esto no es así, ya que hubo una suerte de negociación implícita a través de ciclos de interacción con cláusulas de contrato didáctico específicas, que terminó instaurando normas particulares. Ahora bien, se tiene aún pendiente sugerir una ruta con cláusulas de contrato mucho más específicas que, en el marco de una interacción, genere un discurso reflexivo de tipo $D_{3}$.

Para finalizar, el estudio realizado permite destacar tres observaciones que proveen información valiosa para el conocimiento del docente o para los programas que forman profesores de matemáticas, y que pueden generar un contexto para abordar el asunto expuesto en las últimas líneas del párrafo anterior: i) Es necesario tener conciencia de que las normas sociomatemáticas y los cambios de discurso no emergen automáticamente como consecuencia de la sensación de cumplimiento de las cláusulas del contrato didáctico. Como se mostró mediante este ejercicio analítico, se requiere llevar a cabo una implementación reiterada de tales cláusulas y tener presente cuáles son los conocimientos que pueden ser abordados momento a momento y cuáles los que pueden desarrollarse a largo plazo. ii) No obstante, no cabe duda de que es absolutamente necesario tener conciencia del sistema de normas que se desea instaurar en un aula de clase, que regule tanto la interacción de los sujetos en sí misma (mediante cláusulas del contrato didáctico) como la 
construcción del conocimiento (a través de las normas sociomatemáticas); para este caso en particular, en relación con los procesos de demostración, el aprendizaje es indicado mediante los discursos que tienen lugar en la construcción colectiva de una demostración y la escritura autónoma de la misma. iii) Se debe tener conciencia de que la réplica en un contexto escolar (educación secundaria) de una innovación en el aula de nivel universitario que ha sido considerada exitosa, aun teniendo en cuenta la edad de los estudiantes, no garantiza que también sea exitosa en tal escenario escolar. Es necesario considerar las particularidades del contexto en el cual se implementa la réplica para evitar posteriores fracasos y planear ajustes que eviten dificultades. Usualmente, tales particularidades se concentran en aspectos del contrato didáctico existente en las situaciones instruccionales cotidianas de los escenarios en los cuales se quiere llevar a cabo la innovación y que deben ser cambiados; por ejemplo, puede estar establecido como contrato didáctico de la clase de secundaria que el tiempo para construir una demostración sea exactamente una sesión de clase o una fracción de la misma. Para el caso de la innovación (llevada a cabo en contexto universitario) existe una flexibilidad temporal, dado que no se tiene un tiempo establecido para construir colectivamente una demostración. Asimismo, en la clase de secundaria puede subyacer un contrato a través del cual la participación de los estudiantes sea totalmente pasiva y su responsabilidad con el aprendizaje se limite a poner atención, escribir en su cuaderno la exposición de un profesor y responder a las preguntas eventuales que hace. En la innovación, seguramente es necesario que los alumnos tengan un rol activo, a través del cual participen en la construcción de la demostración y su escritura autónoma. La descripción de estos ejemplos, ilustrados con los análisis elaborados, inducen a pensar que se debe tener conciencia de las cláusulas del contrato existentes en las situaciones instruccionales del contexto en donde se quiere replicar una innovación para que, en una etapa posterior de adaptación de dicha innovación, se tenga un insumo para prever maneras de acoplar Tareas Nuevas (y sus respectivas cláusulas del contrato) que conduzcan a los resultados deseados. 


\section{REFERENCIAS}

Baccaglini-Frank, A. \& Mariotti, M. (2010). Generating Conjectures in Dynamic Geometry: The Maintaining Dragging Model. International Journal of Computers for Mathematical Learning, 15(3), 225-253.

Boero, P. (1999). Argumentation and Mathematical Proof: A Complex, Productive, Unavoidable Relationship in Mathematics and Mathematics Education. Iternational Newsletter on the Teaching and Learning of Mathematical Proof. Recuperado de http://www.lettredelapreuve.org/OldPreuve/Newsletter/990708Theme/990708ThemeUK.html.

Boero, P., Douek, N., Morselli, F. \& Pedemonte, B. (2010). Argumentation and Proof: A Contribution to Theoretical Perspectives and their Classroom Implementation. In M. M. Pinto \& T. F. Kawasaky (Ed.), Proceedings of the 34th Conference of the International Group for the Psychology of Mathematics Education. 1, pp. 179-204. Belo Horizonte, Brazil: PME.

Cobb, P., Boufi, A., McClain, K. \& Whitenack, J. (1997). Reflective Discourse and Collective Reflection. Journal for Research in Mathematics Education, 28(3), 258-277.

Godino, J. D., Font, V., Wilhelmi, M. R. \& Castro, C. d. (2009). Aproximación a la dimensión normativa en Didáctica de la Matemática desde un enfoque ontosemiótico. Enseñanza de las Ciencias, 27(1), 59-76.

Hanna, G. \& de Villiers, M. (2012). Proof and Proving in Mathematics Education. New York: Springer.

Jones, K. \& Herbst, P. (2012). Proof, Proving, and Teacher-Student Interaction: Theories and Contexts. In G. Hanna \& M. de Villiers, Proof and Proving in Mathematics Education (pp. 261-278). New York: Springer.

Krummheuer, G. (1995). The Ethnography of Argumentation. In P. Cobb \& H. Bauersfeld, The Emergence of Mathematical Meaning: Interaction in Classroom Cultures (pp. 229-269). Hillsdale, New Jersey: Lawrence Erlbaum Associates.

Mariotti, M., Bartolini Bussi, M., Boero, P., Ferri, F. \& Garuti, R. (1997). Approaching Geometry Theorems in Contexts: From History and Epistemology to Cognition. In E. Pehkonen, Proceedings of the 21st Conference of the International Group for the Psychology of Mathematics Education (Vol. 1, pp. 180-195). Lahti, Finland: PME.

Martin, T., McCrone, S., Bower, M. \& Dindyal, J. (2005). The Interplay of Teacher and Student Actions in the Teaching and Learning of Geometric Proof. Educational Studies in Mathematics, 60(1), 95-124. 
Mejia-Ramos, J., Fuller, E., Weber, K., Rhoads, K. \& Samkoff, A. (2012). An Assessment Model for Proof Comprehension in Undergraduate Mathematics. Educational Studies in Mathematics, 79(1), 3-18.

Molina, O., Samper, C. \& Perry, P. (2015). Enunciado de un Teorema: ¿Único componente de su significado? Actas XIX Jornadas Nacionales de Educación Matemática: XIX JNEM 2015 (pp. 651-654). Villarrica, Chile: Pontificia Universidad Católica de Chile.

Pedemonte, B. (2007). How Can the Relationship between Argumentation and Proof be Analysed? Educational Studies in Mathematics, 66(1), 23-41.

Perry, P., Samper, C., Molina, O., Camargo, L. \& Echeverry, A. (2012). La geometría del ángulo desde otro ángulo: Una aproximación metodológica alternativa. Épsilon Revista de Educación Matemática, 29(3), 42-56.

Samper, C., Camargo, L., Molina, Ó. \& Perry, P. (2013). Instrumented Activity and Semiotic Mediation: Two Frames to Describe the Conjecture Construction Process as Curricular Organizer. In A. M. Lindmeier \& A. Heinze, Proceedings of the 37th Conference of the International Group for the Psychology of Mathematics Education (Vol. 4, pp. 145-152). Kiel: PME.

Samper, C., Perry, P., Camargo, L., Sáenz-Ludlow, A. \& Molina, O. (2015). A Dilemma that Underlies an Existence Proof in Geometry. Educational Studies in Mathematics, 93(1), 35-50.

Samper, C. \& Plazas, T. (2017). Tipos de mensajes del profesor durante la producción de una demostración en geometría. Educación Matemática, 29(1), 37-60.

Steffe, L. P. \& Thompson, P. W. (2000). Teaching Experiment Methodology: Underlying Principles and Essential Elements . In A. E. Kelly \& R. A. Lesh, Handbook of Research in Mathematics and Science Education (pp. 267-306). Mahwah, EUA: Lawrence Erlbaum Associates.

Stylianides, A. (2007). Proof and Proving in School Mathematics. Journal for Research in Mathematics Education, 38(3), 289-321.

Toulmin, S. (2003). The Uses of Arguments (Actualización de 1a ed.). Cambridge: Cambridge University Press.

Yackel, E. \& Cobb, P. (1996). Sociomathematical Norms, Argumentation, and Autonomy in Mathematics. Journal for Research in Mathematics Education, 27(4), 458-477. 